\title{
The Correct Localization of Borealin in Midbody during Cytokinesis Depends on IQGAP1
}

\author{
Reziya Wumaier $\mathbb{D},{ }^{1,2}$ Abudunaibi Aili, ${ }^{3}$ Hexige Saiyin, ${ }^{1}$ Pingzhao Zhang, ${ }^{1}$ Lihuan Cao $\mathbb{D},{ }^{1}$ \\ and Aikeremujiang Muheremu $\mathbb{D}^{4}$
}

${ }^{1}$ State Key Laboratory of Genetic Engineering, Institute of Genetics, School of Life Sciences, Fudan University, Shanghai 200433, China

${ }^{2}$ Key laboratory of Medical Molecular Virology, Institute of Biomedical Sciences and Institute of Medical Microbiology, Shanghai Medical College, Fudan University, Shanghai 200032, China

${ }^{3}$ Department of Spine Surgery, Sixth Affiliated Hospital of Xinjiang Medical University, Urumqi, Xinjiang 86830001, China

${ }^{4}$ Department of Orthopaedics, Peking University Third Hospital, Beijing 86100191, China

Correspondence should be addressed to Lihuan Cao; lihuancaofdu@gmail.com and Aikeremujiang Muheremu; taklimakan321@163.com

Received 23 March 2020; Revised 31 May 2020; Accepted 8 June 2020; Published 25 June 2020

Academic Editor: Yun-Peng Chao

Copyright (c) 2020 Reziya Wumaier et al. This is an open access article distributed under the Creative Commons Attribution License, which permits unrestricted use, distribution, and reproduction in any medium, provided the original work is properly cited.

Borealin is a key component of chromosomal passenger complex, which is vital in cytokinesis. IQ domain-containing GTPaseactivating protein 1 (IQGAP1) also participates in cytokinesis. The correlation between Borealin and IQGAP1 during cytokinesis is not yet clear. Here, we used mass spectrometry and endogenous coimmunoprecipitation experiments to investigate the interaction between IQGAP1 and Borealin. Results of the current study showed that Borealin interacted directly with IQGAP1 both in vitro and in vivo. Knockdown of IQGAP1 resulted in an abnormal location of Borealin in the midbody. Knocking down Borealin alone, IQGAP1 alone, or Borealin and IQGAP1 at the same time inhibited the completion of cytokinesis and formed multinucleated cells. Our results indicated that IQGAP1 interacts with Borealin during cytokinesis, and the correct localization of Borealin in the midbody during cytokinesis is determined by IQGAP1, and IQGAP1 may play an important role in regulating Borealin function in cytokinesis.

\section{Introduction}

Cytokinesis controls the proper distribution of the replicated cytoplasm and nuclear material in two daughter cells. If this process is abnormal or defective, it will lead to the failure of previous mitotic events and cause cell polyploidy and chromosome instability [1]. In animal cells, cytokinesis usually begins at the anaphase and ends with the complete separation of two daughter cells. After entering the anaphase, the mitotic spindle recompositions to a series of interlaced and antiparallel microtubules, which are called central spindle microtubules. Signals from astral microtubules and central spindle microtubules in centrosome stimulate cells to form a cleavage furrow on the division plane [2]. After the cleavage furrow is formed, the central spindle midzone is reconstructed to form a midbody. The midbody provides an important platform for recruiting and organizing crucial proteins that regulate the detachment of two daughter cells [3]. These midbody proteins can be divided into three subgroups according to their positions: the bulge, the dark zone, and the flanking zone proteins [4].

In the process of cytokinesis, cytoskeleton and many signal proteins need to coordinate to control the dynamic events from the determination of the division plane to the final abscission of two daughter cells. Those proteins include chromosomal passenger complex (CPC), microtubule-associated protein (MAP), and at least three kinesin-like motors, such as KIF4A and KIF20A. Among them, the CPC is mainly 
composed of Aurora B, INCENP, Borealin, and survivin. At different stages during mitosis, three nonkinase subunits of CPC, Borealin, INCENP, and survivin control the targeting of CPC by directly binding with unique proteins, while Aurora B can phosphorylate different substrates [5]. In the metaphase, CPC is located in the centromere and regulates the arrangement and separation of chromosomes [6]. In the anaphase, CPC activates KIF4A through Aurora B and inhibits microtubule depolymerization enzyme KIF2A and stabilizes the central spindle [7, 8]. During abscission, CPC is located in the midbody $[4,9]$, and Aurora B kinase phosphorylates the subunit CHMP4C of endosomal sorting complex required for transport- III (ESCRT III) and negatively regulates the process of two daughter cell detachment [1012]. Interestingly, Borealin is the direct interaction protein between the ESCRT-III subunits Shrb and CHMP4C in Drosophila melanogaster and humans [13, 14]. However, molecular evidence on how CPC regulates the detachment of two daughter cells is still lacking.

Members of the IQ domain-containing GTPase-activating protein (IQGAP) family have four calmodulin binding IQ motifs and a GAP domain for RAS signal transduction [15]. However, those GAP domains do not have a GAP activity due to the lack of arginine finger [16]. In addition to GAP domains, other domains in IQGAPs can also mediate interactions with other proteins [17-19]. IQGAP family proteins exist in many eukaryotes, including Dictyostelium, yeast, Caenorhabditis elegans, and mammals [20]. The IQGAP homologue Rng2 in Schizosaccharomyces pombe and qg1/Cyk1 in Saccharomyces cerevisiae is located in the contractile ring of cells and is essential for cytokinesis [21, 22]. IQGAP homologous protein pes-7 in nematode (C. elegans) also plays important role in cytokinesis [23]. In mammals, three members of the IQGAP family (IQGAP1, IQGAP2, and IQGAP3) have been reported to be essential in cellular activities such as exocytosis, neuronal morphogenesis, cell adhesion, and cell migration [21, 24, 25]. They are also the key regulators in mediating Rho family GTPases and cytoskeleton reorganization. Previous studies have shown that IQGAP1 stabilizes the active forms of RAC1 and CD42 [26, 27] IQGAP family is closely linked to small GTPase network; IQGAP1 interacts with several small GTPases and their network proteins [28]. IQGAP1 seems to accumulate at the contractile ring and activate RhoA in the equatorial cortex [29]. The expression of IQGAP1 mutant in HeLa cells resulted in the formation of multinucleated cells [30]. In the current study, we hypothesized that IQGAP1 interacts with Borealin during cytokinesis and found that IQGAP1 colocalized with Borealin in the midbody, and the correct location of Borealin in the midbody depends on the interaction with IQGAP1.

\section{Materials and Methods}

2.1. Cell Culture. HeLa cells transfected with pCIN4-FlagHA-Borealin and HeLa cells transfected with the control vector were cultured in DMEM containing 10\% fetal bovine serum $(\mathrm{FBS})$ at $37^{\circ} \mathrm{C}$ and $5 \% \mathrm{CO}_{2}$.
2.2. Plasmid Constructs and siRNA Fragments. Full-length Borealin cDNA (CDCA8) was kindly provided by Dr. Ulrike Gruneberg (University of Oxford) and cloned into pCIN4Flag-HA and pCMV-HA/Myc/His vectors. Human EGFPC1-IQGAP1 was provided by David B Sacks from the National Institutes of Health and then cloned into pGex$4 \mathrm{~T}-1$ vector. The siRNA target sequences in the sense orientation were as follows:

IQGAP1-1: 5' - GGAUGAAGCCGCAUUACAdTdT-3'; IQGAP1-2: $5^{\prime}$ - GCUGAAAUUCAAGGCAAUdTdT-3'; Borealin-1: $5^{\prime}$ - CGGAGAGAGCCUGCGAUUAUUdT dT-3';

Borealin-2: $5^{\prime}$-CCUGGAUAUCACCGAAAUAAAdTd $\mathrm{T}-3^{\prime}$;

siControl: $5^{\prime}$-CGUACGCGGAAUACUUCGAdTdT-3'.

2.3. Cell Transfections. Cells were seeded at $40-50 \%$ confluence. For DNA transfections, cells were transfected with $0.3 \mu \mathrm{g}$ and $0.6 \mu \mathrm{g}$ of the indicated plasmid DNA per well, using Lipofectamine 2000 (Invitrogen). For siRNA analyses, cells were transfected with $0.05 \mu \mathrm{M}$ of siRNA oligos using Lipofectamine RNAiMAX (Invitrogen).

2.4. Immunoprecipitation and Western Blotting. HeLa cells were washed with PBS, then lysed in ice-cold BC100 lysis buffer for $20 \mathrm{~min}$ on ice. After centrifugation at $13,000 \mathrm{rpm}$ for $20 \mathrm{~min}$ at $4^{\circ} \mathrm{C}$, the supernatant was incubated with antibody at $4^{\circ} \mathrm{C} 12 \mathrm{~h}$, washed six times with BC100 buffer, and then incubated with protein $\mathrm{A} / \mathrm{G}$ beads for $4 \mathrm{~h}$ at $4^{\circ} \mathrm{C}$. After six washes with $\mathrm{BC} 100$ buffer, cell lysates or immunoprecipitates were subjected to SDS-PAGE and transferred to nitrocellulose membranes. The membranes were first blocked with Tris-buffered saline (TBS, $\mathrm{pH} 7.4$ ) containing $0.1 \%$ Tween-20 and 4\% nonfat milk, and then incubated with primary antibody for at $4^{\circ} \mathrm{C} 12 \mathrm{~h}$, and lastly incubated with secondary antibody for $3 \mathrm{~h}$ at $4^{\circ} \mathrm{C}$. And then, ECL chemiluminescence system (Santa Cruz) was used for detecting proteins.

2.5. Antibodies. Commercially available antibodies were as follows: anti-Borealin (Santa Cruz Biotechnology) $10 \mu \mathrm{g} / \mathrm{mL}$ for western blot and immunofluorescence $5 \mu \mathrm{g} / \mathrm{mL}$, antiIQGAP1 (Santa Cruz Biotechnology) $1 \mu \mathrm{g} / \mathrm{mL}$ for western blot and immunofluorescence $2 \mu \mathrm{g} / \mathrm{mL}$, anti-Aurora B (BD Biosciences) $6 \mu \mathrm{g} / \mathrm{mL}$ for immunofluorescence, anti- $\alpha$-tubulin (Abcam) $5 \mu \mathrm{g} / \mathrm{mL}$ for immunofluorescence, anti- $\beta$-tubulin (Abcam) $5 \mu \mathrm{g} / \mathrm{mL}$ for immunofluorescence, and anti- $\beta$-actin (AC-74, Sigma) $0.1 \mu \mathrm{g} / \mathrm{mL}$ for western blot.

2.6. Protein Complex Purification. HeLa cells were transfected with pCIN4-Flag-HA-Borealin constructs and selected for 3 weeks in $1.5 \mathrm{mg} / \mathrm{mL}$ G418. The tagged Borealin protein levels were detected by western blot. The stable cell lines that express Flag-HA-Borealin were chosen to expand. For protein complex purification, the HeLa/Borealin cells were lysed in BC100 buffer (20 mM Tris-Cl, pH 7.5, 20\% glycerol, $100 \mathrm{mM} \mathrm{NaCl}$, and $0.2 \mathrm{mM}$ EDTA) containing $0.1 \%$ Triton $\mathrm{X}-100$ and protease inhibitor on ice for $4 \mathrm{~h}$. After centrifugation for $40 \mathrm{~min}$ with $12500 \mathrm{rpm}$ at $4^{\circ} \mathrm{C}$, the supernatant of the 


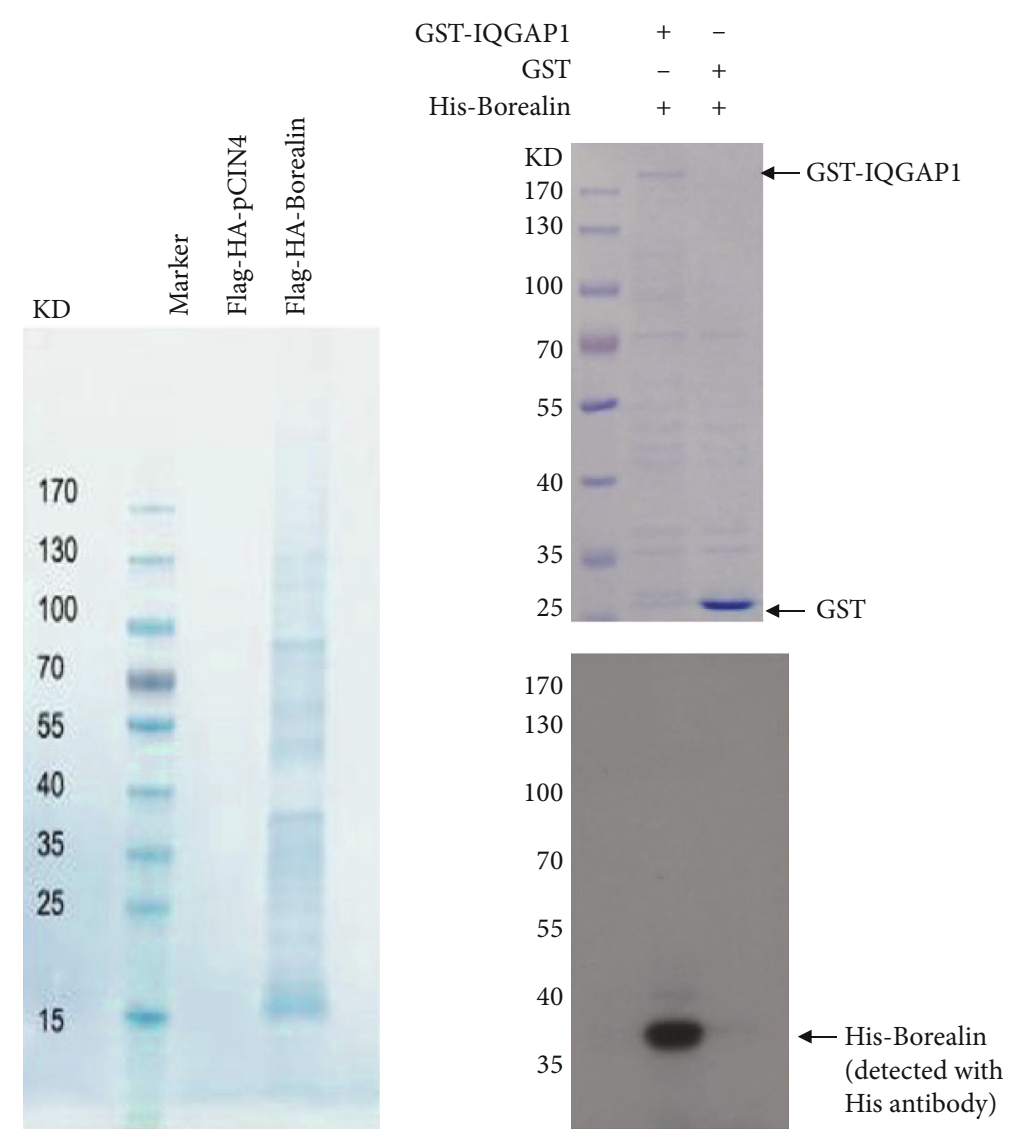

(a)

(b)

His-Borealin

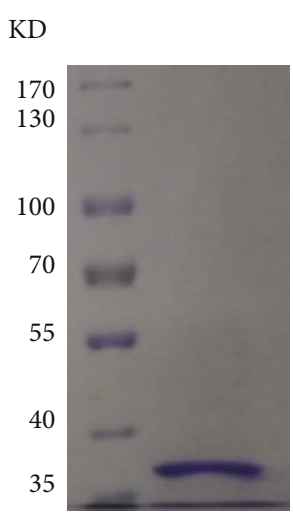

(c)
His-Borealin

Coomassie Blue Staining
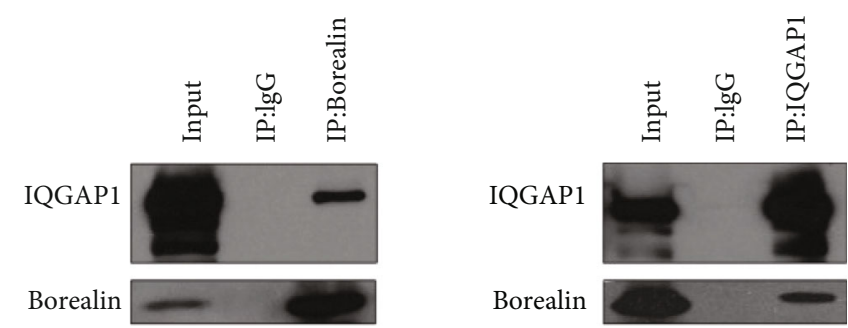

(d)

FIgURE 1: IQGAP1 interacts with Borealin both in vivo and in vitro. (a). Tandem affinity purification of Borealin-containing protein complexes was conducted using HeLa cells stably expressing Flag-HA- (FH-) Borealin and the control group with an empty vector. The protein complex was separated by SDS-PAGE and visualized by Coomassie Blue (CB) staining. The proteins and the number of peptides identified by mass spectrometry are shown in Table 1. (b) Purified His-Borealin from bacteria was incubated with GST-IQGAP or GST that was prebound to glutathione agarose beads. Half of the binding proteins from these beads were separated by SDS-PAGE and visualized by $\mathrm{CB}$, and half were detected by western blotting with anti-His antibody. (c) Purified His-Borealin was separated by SDS-PAGE and visualized by CB. (d) The endogenous Borealin was immunoprecipitated by anti-Borealin antibody, and the immunoprecipitates were detected by western blotting with the indicated antibodies. Endogenous IQGAP1 was immunoprecipitated by anti-IQGAP1 antibody, and the immunoprecipitates were detected by western blotting with the indicated antibodies. Three independent biological replicates were performed in the purification of recombinant protein and immunoprecipitation experiments. 
cell lysates was immunoprecipitated by anti-Flag antibodyconjugated M2 agarose (Sigma). After washing three times with the BC100 buffer to remove the unbounded protein, immunoprecipitated by anti-HA antibody-conjugated M2 agarose (Sigma), the bound polypeptides were eluted with peptide and resolved by SDS-PAGE for Coomassie Blue staining. Gel lanes (strips) were subjected to mass spectrometric sequencing.

2.7. Purification of Recombinant Protein. The recombinant GST-IQGAP or His- Borealin was expressed in Escherichia coli strain BL21-CodonPlus (DE3)-RIL with $0.5 \mathrm{mM}$ IPTG (isopropyl- $\beta$-D-thiogalactopyranoside) at $23^{\circ} \mathrm{C}$ after 12 hour and 16-hour induction, respectively. Cells were collected and lysed, and the GST recombinant protein and His recombinant protein were purificated as previously described [31].

2.8. GST Pull-Down Assay. GST-IQGAP protein was immobilized on Glutathione Sepharose 4B (Amersham Biosciences). After washing with pull-down buffer $(25 \mathrm{mM}$ Tris- $\mathrm{HCl}, \mathrm{pH} 7.5,10 \%$ glycerol, 0.1\% NP-40,150 mM NaCl, $1 \mathrm{mM}$ EDTA, $1 \mu \mathrm{g} / \mathrm{mL}$ leupeptin, and $1 \mathrm{mM}$ DTT), the beads were incubated with recombinant His-borealin for $3 \mathrm{~h}$ at $4^{\circ} \mathrm{C}$. The beads were then washed six times with a binding buffer. The bound proteins were then resuspended in sample buffer for SDS-PAGE and western blot analysis.

2.9. Immunofluorescence and Confocal Microscopy. For immunofluorescence tests, cells were plated on chamber slides and fixed with $4 \%$ paraformaldehyde for $25 \mathrm{~min}$ at $23^{\circ} \mathrm{C}$. To examine the immunofluorescence of protein at each mitotic stage, cells were synchronized by double-thymidine block and release to fresh media. A staging system was used to identify the different phases of mitosis based on the DNA and extent of chromosome alignment and separation, which was described in our previous publication [32]. For example, when after double-thymidine block cells were released by fresh media, they entered the $M$-phase, and 8 hours after releasing the cells into fresh media, they reached cytokinesis [32]. After fixation, cells were permeabilized with $0.2 \%$ Triton for $5 \mathrm{~min}$, blocked with $5 \%$ goat serum in PBS. The cells were incubated with primary antibody solutions at $4^{\circ} \mathrm{C}$ overnight. Slides were washed, incubated with fluorescence-tagged secondary antibodies (Alexa Fluor 488, 568, Molecular Probes, Invitrogen), and counterstained with DAPI (Vector Labs) for $1 \mathrm{~h}$ at $4^{\circ} \mathrm{C}$. Images were acquired using a Zeiss LSM710 confocal microscope equipped with a 60x objective. Fluorescence from multiple planes throughout the cells were collected to create a stack of two-dimensional (2D) images ( $z$-stack); each plane showing fluorophore localization on the $x$ and $y$ planes; those planes were computationally reconstructed to form the $3 \mathrm{D}$ image of the cells, which were then projected as a $2 \mathrm{D}$ view on a computer screen [33]. For time-lapse imaging, Zeiss TRIF fluorescent live cell imaging microscope equipped with a $20 \mathrm{x}$ objective was used. Images of proteins of interest were acquired using identical imaging settings. Image processing and figures were made using PhotoShop CS (Adobe).
TABLE 1: Summary of mass spectrometry results from the affinity purification of Flag-HA-tagged Borealin. Several proteins identified from the affinity purifications are listed along with their relative Mascot score and number of peptides.

\begin{tabular}{lcc}
\hline Proteins & Score & Peptides \\
\hline Borealin & 4462 & 19 \\
GRP78 & 4105 & 35 \\
IQGAP1 & 3006 & 27 \\
ATBP & 2093 & 18 \\
KIF11 & 2091 & 26 \\
BIRC5 & 522 & 5 \\
INCENP & 371 & 8 \\
AURKB & 85 & 2 \\
\hline
\end{tabular}

\section{Results}

3.1. Borealin Interacts with IQGAP1 Both In Vitro and In Vivo. The HeLa monoclonal cell line was constructed with stably expressing Flag-HA-tagged Borealin protein, after treatment with double-thymidine block (DTB); cells whose division process synchronized to cytokinesis were collected. After tandem affinity purification (Figure 1(a)), the complex formed by Borealin protein was analyzed by mass spectrometry. We identified IQGAP1 as one of the Borealininteracting proteins, and its score was comparable to or higher than the other members of CPC, survivin, INCENP, and Aurora B (Table 1).

IQGAP1 is an important component in a small GTPase network and plays a key role in cytokinesis. To find the relationship between IQGAP1 and Borealin in cytokinesis, we first investigated whether the interaction between IQGAP1 and Borealin is direct. Recombinant GST-IQGAP1 protein and His-Borealin protein were expressed and purified, respectively, in bacteria. As shown in Figure 1(b) and 1(c), GST-IQGAP1, but not single GST, binds to His-Borealin in the pulled-down complex, indicating that IQGAP1 and Borealin interact physically. Then, we used endogenous immunoprecipitation to detect whether IQGAP1 interacted with Borealin in HeLa cells. As shown in Figure 1(d), Borealin was detected in anti-IQGAP1 immunoprecipitates by western blotting, and IQGAP1 was also detected in antiBorealin immunoprecipitates. Therefore, our data demonstrate that Borealin directly interacts with IQGAP1 both in vitro and in vivo.

3.2. IQGAP1 Colocalized with Borealin and Aurora $B$ in the Midbody. We then observed the subcellular localization of Borealin and IQGAP1 at different stages of HeLa cell division by two-dimensional immunofluorescence assay analysis. We found that Borealin changed its position during cell cycle progression. In the interphase, Borealin was found in the nucleus; in the prophase, Borealin was enriched in the centromere; in the metaphase, it is mainly located in the equator and centromere; in the anaphase, it was mainly located in the central spindle area and cleavage furrow (Figure 2(a)). Borealin was located in the arm of the midbody during the 


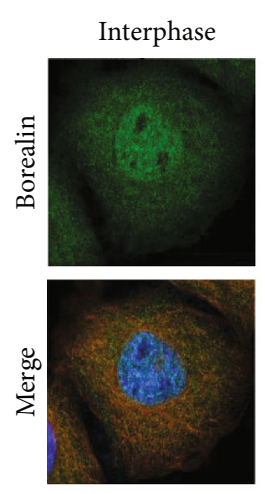

Telophase
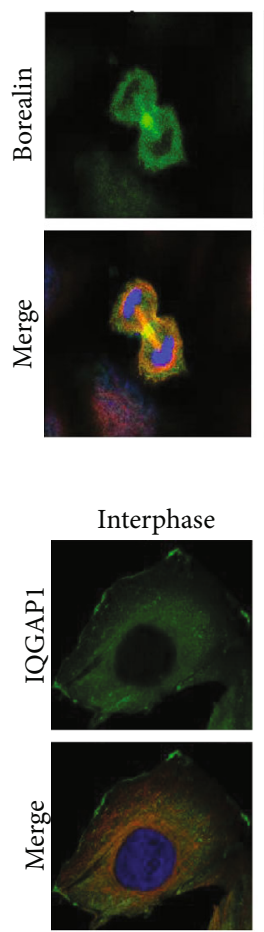

Telophase
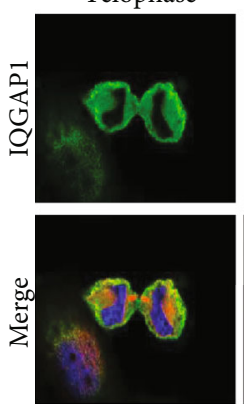

Prophase
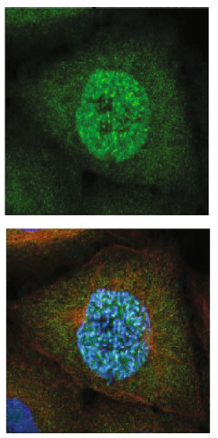

Ingression
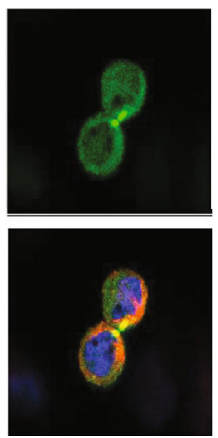

(a)
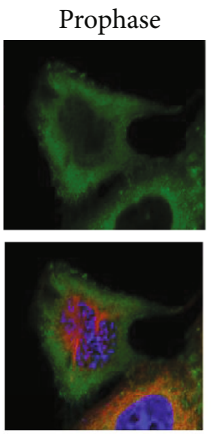

Ingression
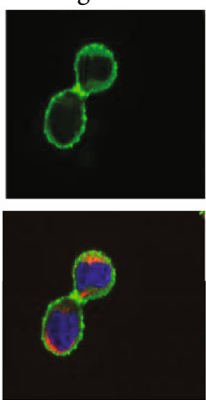

Metaphase
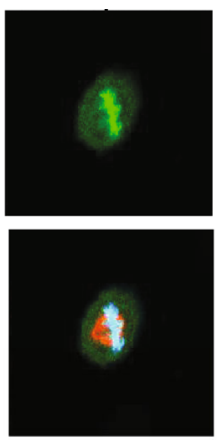

Abscission
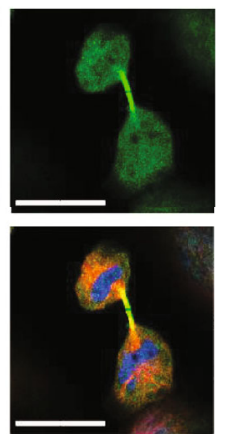

Borealin (green)

$\alpha$-Tubulin (red)

DAPI (blue)
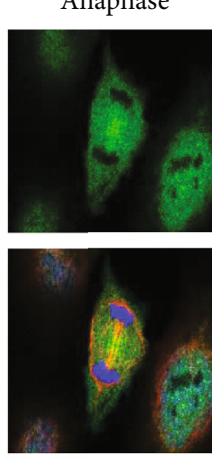

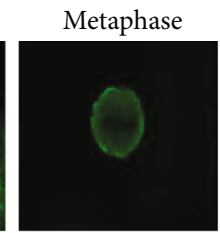

Anaphase
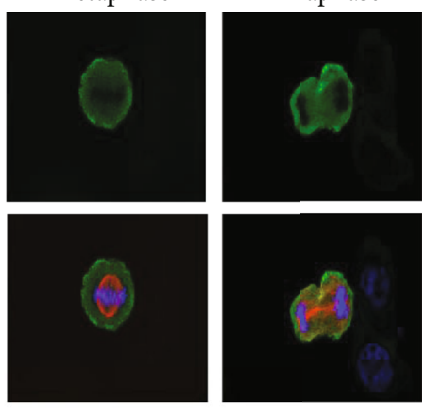

Abscission
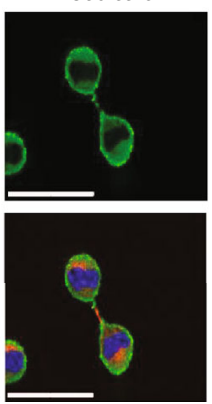

IQGAP1 (green)

$\alpha$-Tubulin (red)

DAPI (blue)

(b)

FIgURE 2: Dynamic localization of Borealin and IQGAP1 during mitosis in HeLa cells. (a) HeLa cells synchronized by double-thymidine treatment were fixed and stained with Borealin antibody (green), alpha-tubulin antibody (red), and DAPI (blue) or (b) IQGAP1 antibody (green) and alpha-tubulin antibody (red), and DAPI (blue). Three independent biological replicates were performed.

formation of the intercellular bridge between the two daughter cells (Figure 2(a)). As for IQGAP1 protein, we found that it was enriched in cell membrane during the interphase, prophase, and metaphase, and it was enriched in the cleavage furrow at the anaphase and telophase. IQGAP1 was also enriched in the midbody during the formation of bridges between two daughter cells (Figure 2(b)).

In view of their coherent enrichment in the cleavage furrow and cytokinesis midbody at later stages of cell division, analyzing the three-dimensional reconstruction images 

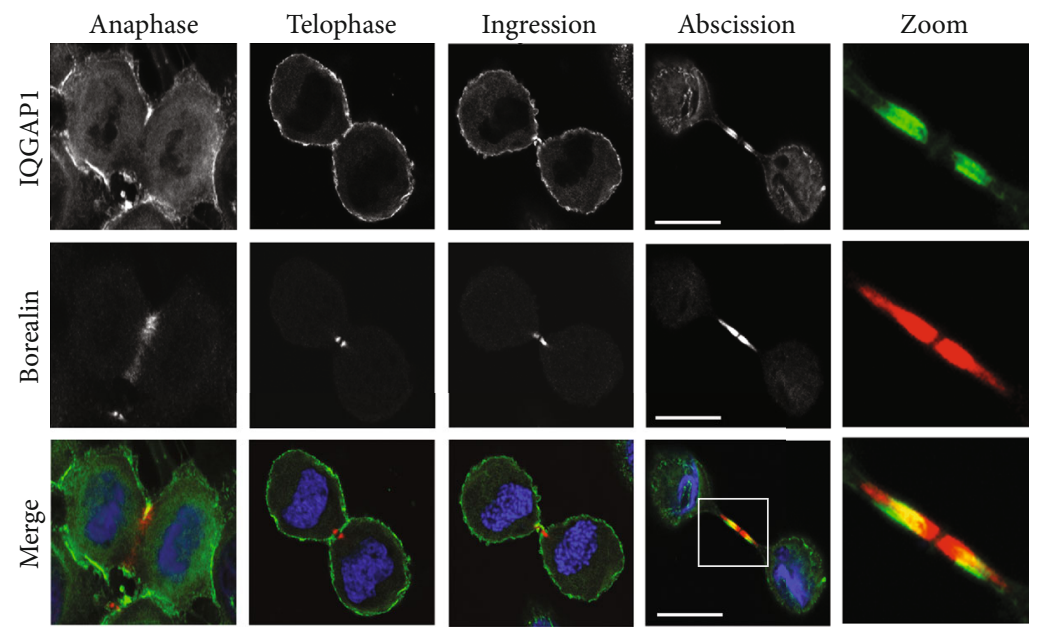

IQGAP1 (green) Borealin (red)

(a)
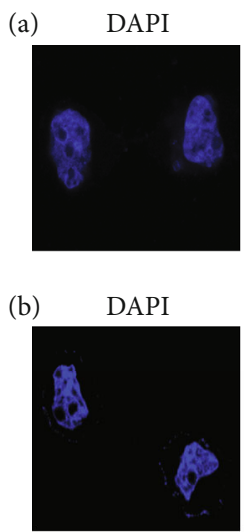

(c)

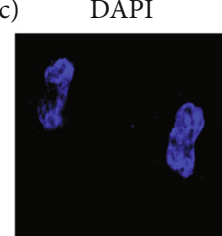

Borealin

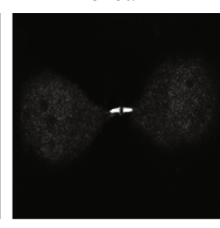

IQGAP1

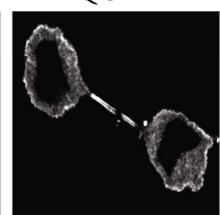

IQGAP1

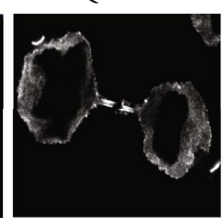

AuroraB

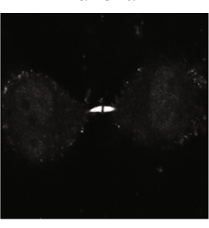

Borealin

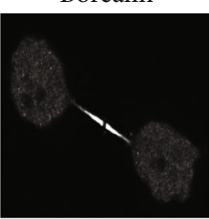

AuroraB

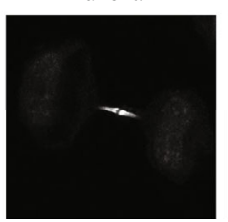
Zoom
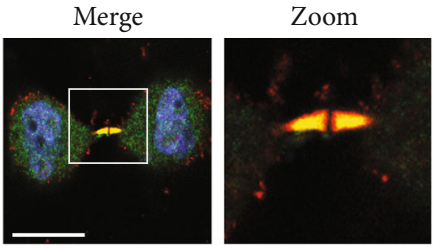

Borealin (green) AuroraB (red)

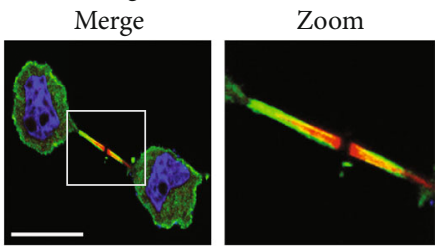

IQGAP1 (green) Borealin (red)
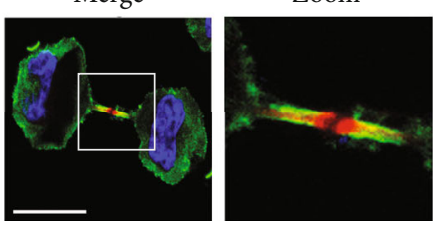

IQGAP1 (green) AuroraB (red)

(b)

Figure 3: IQGAP1 colocalized with Borealin and Aurora B at the midbody.(a) HeLa cells synchronized by double-thymidine treatment were fixed and stained with IQGAP1 antibody (green), Borealin antibody (red), and DAPI (blue). (b) Colocalization of IQGAP1, Borealin, and Aurora B at the midbody. (A) HeLa cells synchronized by double-thymidine treatment were fixed and stained with Borealin antibody (green), Aurora B antibody (red), and DAPI (blue); or (B) IQGAP1 antibody (green), Borealin antibody (red), and DAPI (blue); or (D) IQGAP1 antibody (green), Aurora B antibody (red), and DAPI (blue). Bar $=10 \mu \mathrm{m}$.

of cells that were projected to two dimensional plane, we noticed the colocalization of Borealin and IQGAP1 at different stages of cell cycle, and immunofluorescence tests indicated that Borealin and IQGAP1 were colocated in the cleavage furrow (Figure 3(a)) and midbody (Figure 3(a)).

We also observed that the colocalization of other CPC members Aurora B and IQGAP1 (Figure 3(b)), Borealin and Aurora B, Borealin and IQGAP1, and IQGAP1 and Aurora $B$ were colocalized in the midbody. Interestingly, Aurora B, Borealin, and IQGAP1 were all located in the flanking region of the midbody. Meanwhile, IQGAP1 was also located more outside of the flank region, not close to the dark area, compared with Aurora B and Borealin. These results of colocalization in the midbody suggest that IQGAP1 with CPC complex may functionally regulate each other during cytokinesis.

3.3. Location of Borealin in Midbody Depends on IQGAP1. In order to study the functional significance of the interaction between Borealin and IQGAP1, we first analyzed the location of IQGAP1 after Borealin was knocked down. We found that Borealin knockdown resulted in the failure of cell division 


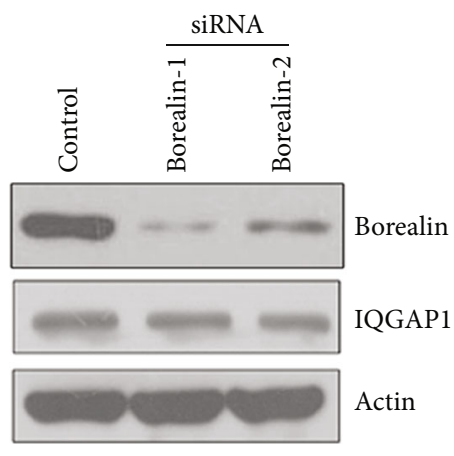

(a)
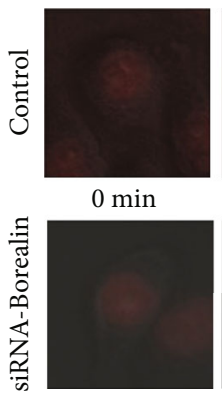

$0 \mathrm{~min}$
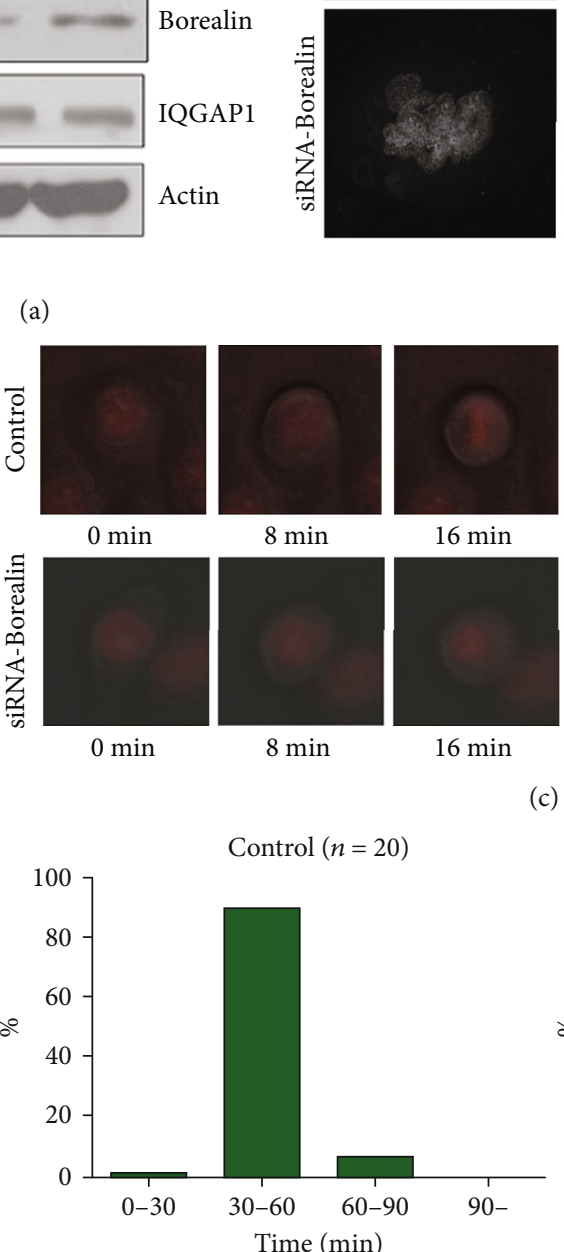
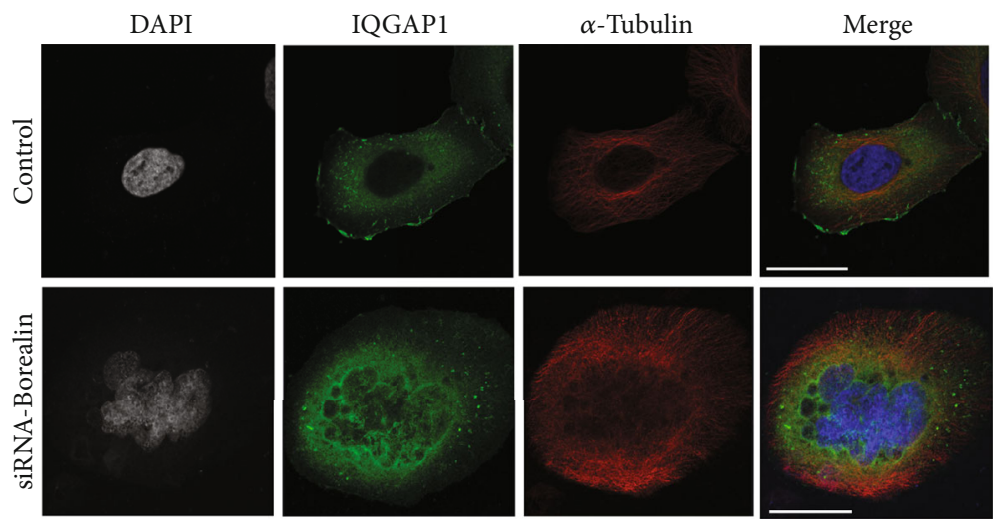

IQGAP1 (green)

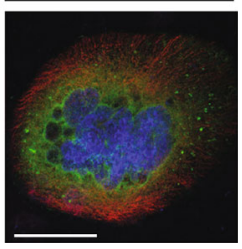

$\alpha$-tubulin (red)

(b)
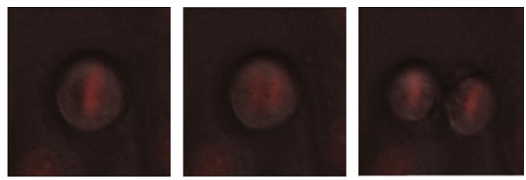

$32 \mathrm{~min}$

$40 \mathrm{~min}$

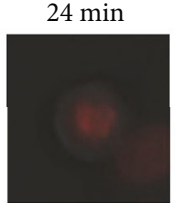

$32 \min$

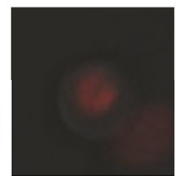

$56 \mathrm{~min}$

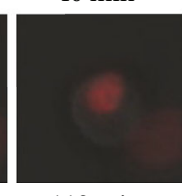

$112 \mathrm{~min}$

(c)

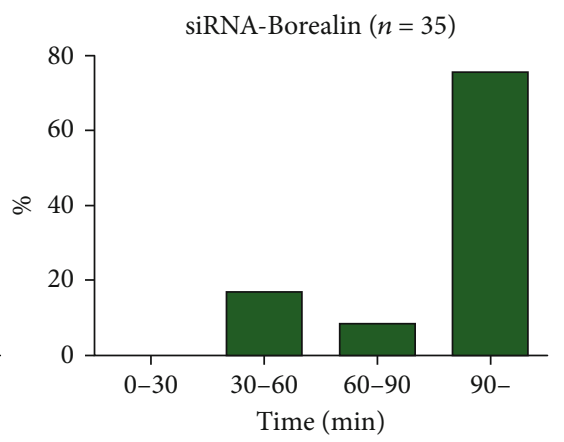

(d)

FIGURE 4: Borealin depletion distorted the cell's architecture. (a). HeLa cells were treated with siRNAs against Borealin or control. After $48 \mathrm{~h}$, cell lysates were detected by western blotting with the indicated antibodies. (b). HeLa cells were treated with siRNAs against Borealin or control. After $48 \mathrm{~h}$, cells were fixed and stained with IQGAP antibody (green), alpha-tubulin antibody (red), and DAPI (blue). Bar $=10$ $\mu \mathrm{m}$. (c). The HeLa cell line with stably expressing mCherry-H2B was treated with siRNAs against Borealin or control. After $48 \mathrm{~h}$, timelapse imaging was used to observe the process of cell division. (d). Statistic diagram of the time required for cells from nuclear envelope breakdown (NEBD) to the anaphase.

from the metaphase to anaphase and the inability of the duplicated sister chromatids to separate, leading to considerable distortions in cell structure (Figure 4). We believe that this is related to the dynamic localization of CPC complexes in the process of cell division and their multiple functions. Therefore, we could not reach a conclusion on the distribution of IQGAP1 during cytokinesis after Borealin was depleted.

When analyzing the location of Borealin after the knockdown of the IQGAP1 protein, we found that the localization of Borealin was disturbed in the midbody. Borealin was located in only half of the flank region close to the dark area, but not in the lateral region of the flank, which was the position of IQGAP1 in the midbody (Figure 5). In the control cells, Borealin was located in the entire flanking region of the midbody. Therefore, it is safe to assume that the correct location of Borealin in the midbody depends on the IQGAP1 protein.

3.4. IQGAP1 May Play an Important Role in Regulating Borealin Function in Cytokinesis. Finally, we studied the functions of Borealin and IQGAP1 in cytokinesis. When 


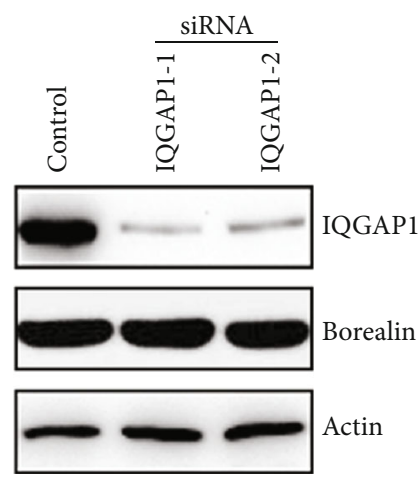

(a)
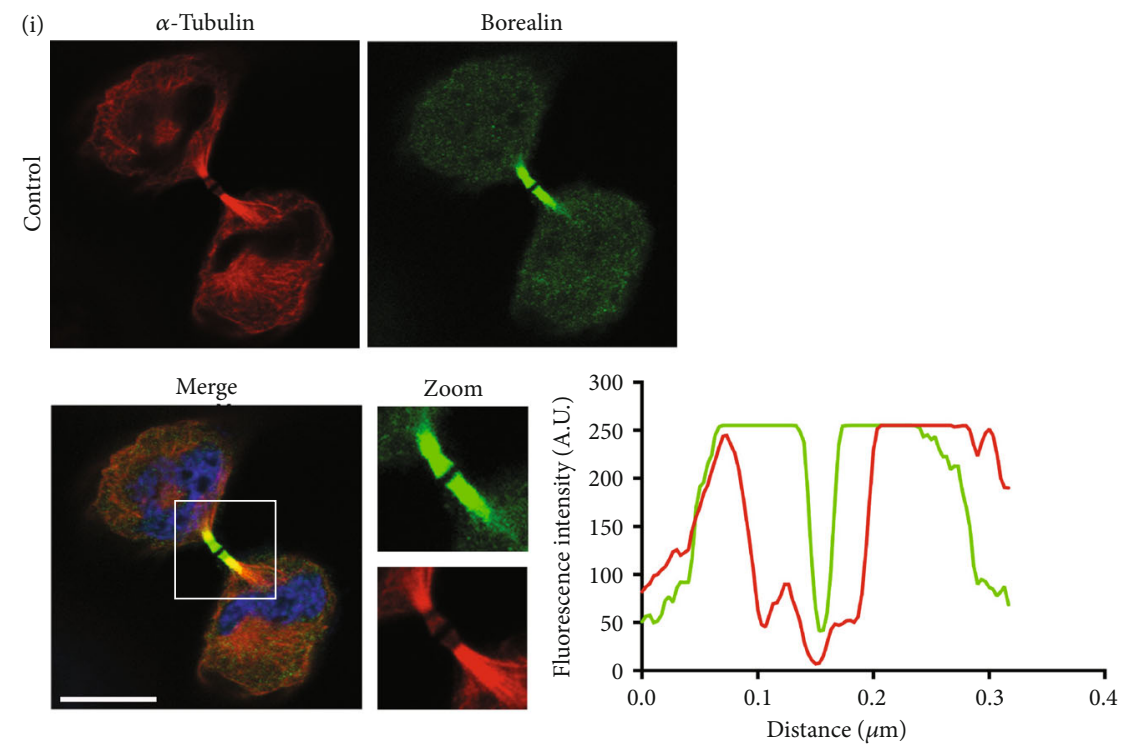

- $\alpha$-Tubulin

— Borealin

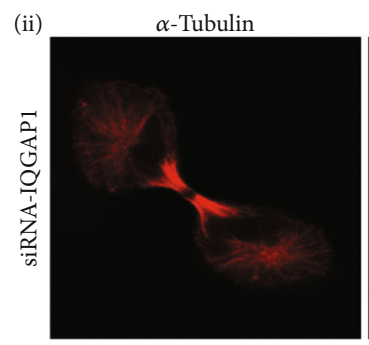

Borealin
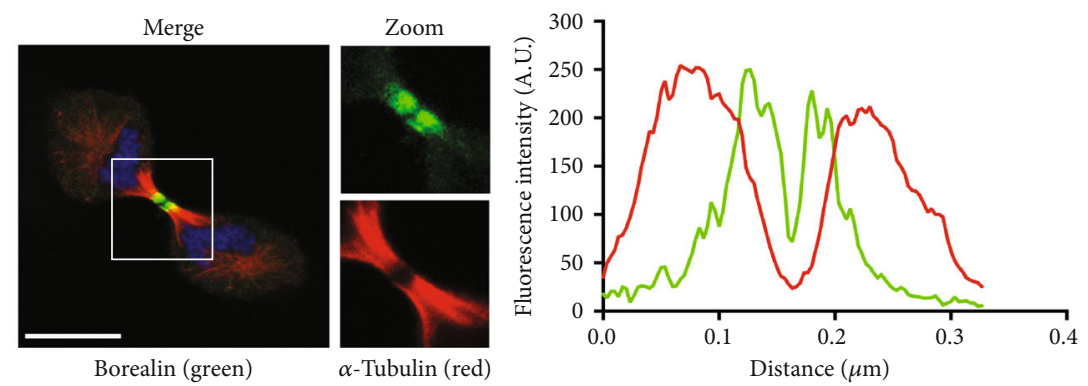

$\alpha$-Tubulin

(b)

Figure 5: Continued. 


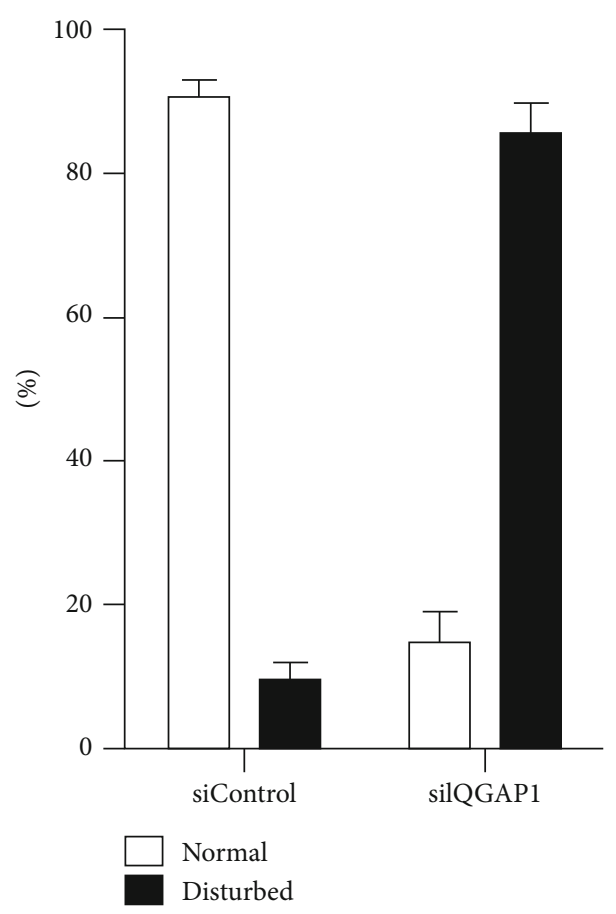

(c)

FIGURE 5: Localization of Borealin in the midbody depends on IQGAP1. (a). HeLa cells were treated with siRNAs directed against IQGAP1 or control. After $48 \mathrm{~h}$, cell lysates were detected by western blotting with the indicated antibodies. (b). HeLa cells were treated with siRNAs direct against IQGAP1 or control. After $48 \mathrm{~h}$, cells were fixed and stained with Borealin antibody (green), alpha-tubulin antibody (red), and DAPI (blue). Bar $=10 \mu \mathrm{m}$. The graph below shows the overlap of fluorescence intensity peaks along profile localization of Borealin on midbody in the merged micrograph. (c) The percentage of Borealin midbody location disturbed in IQGAP1-depleted cells and in control cells. A minimum of 150 cells were counted per sample in three independent experiments. Error bars represent \pm S.E.

Borealin or/and IQGAP1 was knocked down in HeLa cells, cytokinesis could not be completed, leading to the formation of multinucleated cells. When we knocked down Borealin using two different siRNA-targeting sequences, it resulted in a $33 \%$ increase in the proportion of polynuclear cells. In the meanwhile, knockdown of IQGAP1 in HeLa cells resulted in a $12 \%$ increase in the percentage of polynuclear cells. When IQGAP1 and Borealin were knocked down together, the percentage of polynuclear cells was 30-35\%, similar to that of Borealin knock down (Figure 6). Borealin depletion results in the failure of entering cytokinesis, and its location during cytokinesis depends on IQGAP1, so these data suggest that IQGAP1 may play an important role in regulating Borealin function in cytokinesis.

\section{Discussion}

Proteomics and cell biology studies have shown that more than 100 proteins are located in the convex, dark, and flanking regions of the midbody. The midbody is vital as a platform for these proteins to regulate the final detachment of two daughter cells $[3,23]$. It has been known that the CHMP4C subunit of the endosomal sorting complex ESCRT-III can be assembled into helical filaments only when the $\mathrm{CPC}$ accumulated in the flanking region of the midbody is removed. This CPC-mediated ESCRT-III regulation is thought to be a cell division checkpoint to prevent the mid- body from splitting and detaching when DNA is present, thus avoiding the formation of genetically abnormal daughter cells. However, molecular evidence lacks on how CPC regulates the detachment of two daughter cells.

Borealin, a member of CPC complex, interacts with subunits CHMP4A, CHMP4B, and CHMP4C of ESCRT-III. Therefore, we constructed a Flag-HA double-tagged and Borealin overexpressing cell line, collected cells whose division process synchronized to the cytokinesis stage, and investigated Borealin-interacting proteins in the cytokinesis process to find the key regulator proteins. We identified IQGAP1 as a Borealin-interacting protein during cytokinesis and found that IQGAP1 colocalized with Borealin in the midbody, and the location of Borealin in the midbody depended on its interaction with IQGAP1.

Aurora B, a member of the CPC complex, phosphorylated CHMP4C during cytokinesis, and high Aurora B activity delays the final detachment $[10,11,34]$ Another study revealed that the interaction between Aurora B function and citron kinase have a synergic effect on cytokinesis [31] It was also found that ANCHR (abscission/no cut checkpoint regulator; ZFYVE19) is an interacting protein of Aurora B and a key regulatory factor for detaching from the checkpoint [35]. In the current study, we also found that Aurora B was colocalized with Borealin-interacting protein IQGAP1, and location of Aurora B in midbody was disrupted when IQGAP1 was knocked down (data not shown). Depletion of 


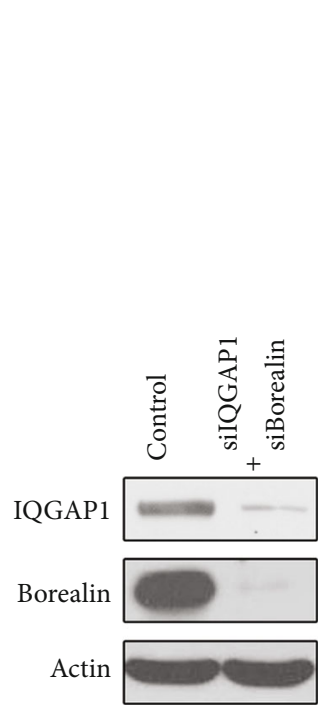

(a)
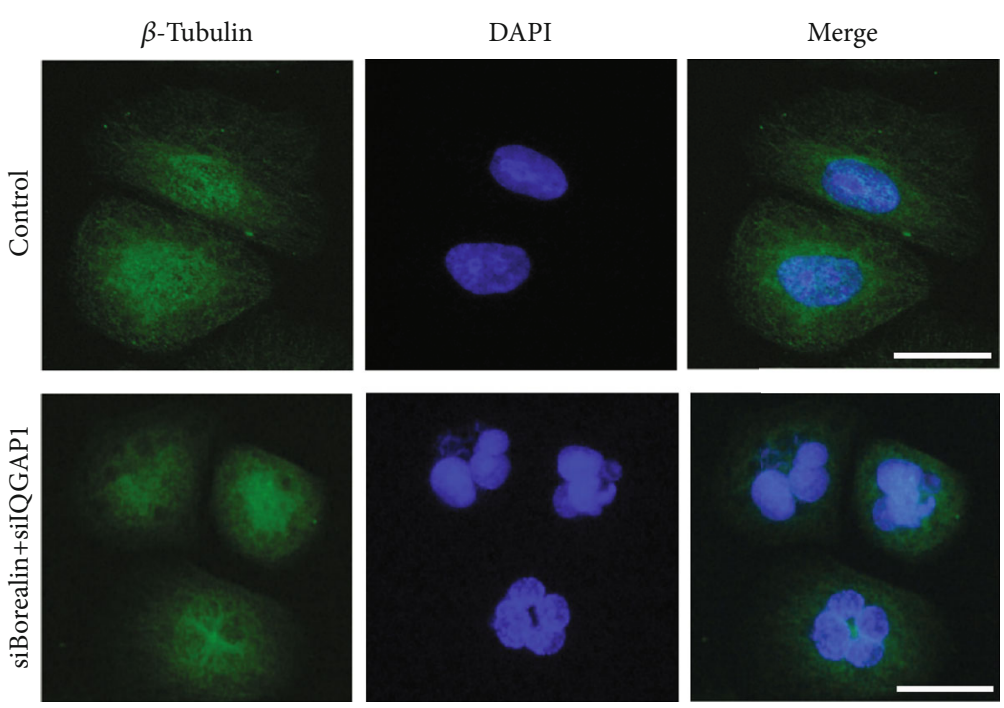

$\beta$-Tubulin (green) DAPI (blue)

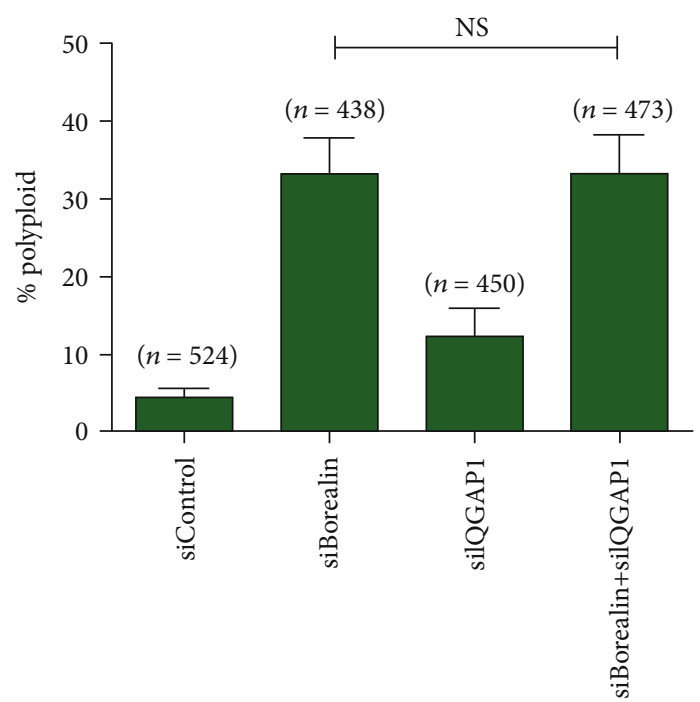

(c)

FIGURE 6: IQGAP1 and Borealin codepletion inhibits cytokinesis completion. (a) HeLa cells were treated with siRNAs directed against Borealin and IQGAP1 together or control. After $48 \mathrm{~h}$, cell lysates were detected by western blotting with the indicated antibodies. (b) RNAi-mediated silencing of Borealin and IQGAP1 together induced multinucleation in HeLa cells. After $48 \mathrm{~h}$, cells were fixed and stained with tubulin antibody (green) and DAPI (blue). (c) IQGAP1 and Borealin codepletion inhibits cytokinesis completion. HeLa cells were individually treated with siRNAs against Borealin alone, IQGAP1 alone, or CDCA8 and IQGAP1 together, and were fixed after $72 \mathrm{~h}$. At least 400 cells were counted every time from three independent experiments. Error bars indicate S.D. Error bars represent \pm S.E. ${ }^{* * *} p<0.001 ;$ n.s., not statistically significant.

IQGAP1 may impare the stability of CPC complex members in the midbody.

Although other members of IQGAP family and IQGAP1 are generally considered to be related to cytokinesis $[29,30]$, the detailed function of other IQGAPs in cytokinesis is not yet clear. Adachi et al. [36] reported that IQGAP3, rather than IQGAP1, is mainly involved in the formation of contractile rings during cytokinesis. Another study proposed that IQGAP1 participated in reassembling nuclear pore complexes during cytokinesis [37]. Our data indicated that IQGAP1 is located in the midbody, while Aurora B and Borealin were all located in the flanking region. This colocaliza- tion at the midbody is presented with CPC components occupying the central region and IQGAP1 decorating halfway down the midbody arms. In our experiments, knocking down Borealin would lead to the failure of transition from the metaphase to anaphase, causing the formation of polynuclear cells. Meanwhile, knocking down IQGAP1 affected the normal location of Borealin in the midbody, then caused the failure of cytokinesis and formed polynuclear cells. While Borealin is the main component of cellular division, IQGAP1 affects cell division by controlling the location of Borealin during the final abscission of two daughter cells. We believe that is the reason why after simultaneously knocking down 
both Borealin and IQGAP1, there was no further increase of polynuclear cells than knocking down Borealin alone.

Our results provide preliminary evidence of functional links between CPC complexes and IQGAP1 during cytokinesis. Results of the current study can be further translated to find drug targets for tumorigenesis and innate immunity [38-40]. Meanwhile, more cell lines are required in the future to further investigate this correlation, and further research is needed to study the role of IQGAP in stabilizing the CPC and the mechanism of how CPC and IQGAP1 play coordinated role during the completion of cytokinesis.

\section{Conclusion}

Our results indicate that IQGAP1 interacts with Borealin during cytokinesis and plays key role in cell division, and the correct localization of Borealin in the midbody is determined by IQGAP1.

\section{Data Availability}

All data generated or analyzed during this study are included in this published article.

\section{Conflicts of Interest}

The authors declare that they have no competing interests.

\section{Authors' Contributions}

RW performed the experiments. YZ, HS, PZ, and LY assisted RW with the experiments. RW, YZ, HS, PZ, and LY analyzed the data. LC designed the experiments and improved the manuscript. All authors read and approved the manuscript and agree to be accountable for all aspects of the research in ensuring that the accuracy or integrity of any part of the work is appropriately investigated and resolved.

\section{Acknowledgments}

This study was funded by the National Natural Science Foundation of China (Grant Nos. 81860235 and 31071186). We are grateful to Ulrike Gruneberg (University of Oxford) for providing the Borealin plasmid, and to David B Sacks (National Institutes of Health) for providing the IQGAP1 plasmid. We would like to thank Professor Gao Qian from the Key Laboratory of Medical Molecular Virology (Fudan University) for his suggestions on the study design and insightful discussions of our study results.

\section{References}

[1] B. Lacroix and A. S. Maddox, "Cytokinesis, ploidy and aneuploidy," The Journal of Pathology, vol. 226, no. 2, pp. 338351, 2012.

[2] P. P. D’Avino, M. G. Giansanti, and M. Petronczki, "Cytokinesis in animal cells," Cold Spring Harbor Perspectives in Biology, vol. 7, no. 4, article a015834, 2015.
[3] B. Mierzwa and D. W. Gerlich, "Cytokinetic abscission: molecular mechanisms and temporal control," Developmental Cell, vol. 31, no. 5, pp. 525-538, 2014.

[4] C. K. Hu, M. Coughlin, and T. J. Mitchison, "Midbody assembly and its regulation during cytokinesis," Molecular Biology of the Cell, vol. 23, no. 6, pp. 1024-1034, 2012.

[5] M. Carmena, M. Wheelock, H. Funabiki, and W. C. Earnshaw, "The chromosomal passenger complex (CPC): from easy rider to the godfather of mitosis," Nature Reviews. Molecular Cell Biology, vol. 13, no. 12, pp. 789-803, 2012.

[6] U. R. Klein, E. A. Nigg, and U. Gruneberg, "Centromere targeting of the chromosomal passenger complex requires a ternary subcomplex of Borealin, survivin, and the $\mathrm{N}$-terminal domain of INCENP," Molecular Biology of the Cell, vol. 17, no. 6, pp. 2547-2558, 2006.

[7] R. N. Bastos, S. R. Gandhi, R. D. Baron, U. Gruneberg, E. A. Nigg, and F. A. Barr, "Aurora B suppresses microtubule dynamics and limits central spindle size by locally activating KIF4A," The Journal of Cell Biology, vol. 202, no. 4, pp. 605621, 2013.

[8] R. Uehara, Y. Tsukada, T. Kamasaki et al., "Aurora B and Kif2A control microtubule length for assembly of a functional central spindle during anaphase," The Journal of Cell Biology, vol. 202, no. 4, pp. 623-636, 2013.

[9] U. Gruneberg, R. Neef, R. Honda, E. A. Nigg, and F. A. Barr, "Relocation of Aurora B from centromeres to the central spindle at the metaphase to anaphase transition requires MKlp2," The Journal of Cell Biology, vol. 166, no. 2, pp. 167-172, 2004.

[10] C. Norden, M. Mendoza, J. Dobbelaere, C. V. Kotwaliwale, S. Biggins, and Y. Barral, "The NoCut pathway links completion of cytokinesis to spindle midzone function to prevent chromosome breakage," Cell, vol. 125, no. 1, pp. 85-98, 2006.

[11] P. Steigemann, C. Wurzenberger, M. H. A. Schmitz et al., "Aurora B-mediated abscission checkpoint protects against tetraploidization,” Cell, vol. 136, no. 3, pp. 473-484, 2009.

[12] J. Mathieu, C. Cauvin, C. Moch et al., "Aurora B and cyclin B have opposite effects on the timing of cytokinesis abscission in Drosophila germ cells and in vertebrate somatic cells," Developmental Cell, vol. 26, no. 3, pp. 250-265, 2013.

[13] L. Capalbo, E. Montembault, T. Takeda, Z. I. Bassi, D. M. Glover, and P. P. D'Avino, "The chromosomal passenger complex controls the function of endosomal sorting complex required for transport-III Snf 7 proteins during cytokinesis," Open Biology, vol. 2, no. 5, p. 120070, 2012.

[14] J. G. Carlton, A. Caballe, M. Agromayor, M. Kloc, and J. Martin-Serrano, "ESCRT-III governs the Aurora Bmediated abscission checkpoint through CHMP4C," Science, vol. 336, no. 6078, pp. 220-225, 2012.

[15] L. Weissbach, J. Settleman, M. F. Kalady et al., "Identification of a human rasGAP-related protein containing calmodulinbinding motifs," The Journal of Biological Chemistry, vol. 269, no. 32, pp. 20517-20521, 1994.

[16] V. B. Kurella, J. M. Richard, C. L. Parke, L. F. Lecour Jr., H. D. Bellamy, and D. K. Worthylake, "Crystal structure of the GTPase-activating protein-related domain from IQGAP1," The Journal of Biological Chemistry, vol. 284, no. 22, pp. 14857-14865, 2009.

[17] M. D. Brown and D. B. Sacks, "IQGAP1 in cellular signaling: bridging the GAP," Trends in Cell Biology, vol. 16, no. 5, pp. 242-249, 2006. 
[18] J. M. Smith, A. C. Hedman, and D. B. Sacks, "IQGAPs choreograph cellular signaling from the membrane to the nucleus," Trends in Cell Biology, vol. 25, no. 3, pp. 171-184, 2015.

[19] A. C. Hedman, J. M. Smith, and D. B. Sacks, "The biology of IQGAP proteins: beyond the cytoskeleton," EMBO Reports, vol. 16, no. 4, pp. 427-446, 2015.

[20] K. B. Shannon, "IQGAP family members in yeast, Dictyostelium, and mammalian cells," International Journal of Cell Biology, vol. 2012, Article ID 894817, 14 pages, 2012.

[21] J. A. Epp and J. Chant, "An IQGAP-related protein controls actin-ring formation and cytokinesis in yeast," Current Biology, vol. 7, no. 12, pp. 921-929, 1997.

[22] K. Eng, N. I. Naqvi, K. C. Y. Wong, and M. K. Balasubramanian, "Rng2p, a protein required for cytokinesis in fission yeast, is a component of the actomyosin ring and the spindle pole body," Current Biology, vol. 8, no. 11, pp. 611-621, 1998.

[23] A. R. Skop, J. Y. Hongbin Liu III, B. J. Meyer, and R. Heald, "Dissection of the mammalian midbody proteome reveals conserved cytokinesis mechanisms," Science, vol. 305, no. 5680, pp. 61-66, 2004.

[24] S. C. Mateer, N. Wang, and G. S. Bloom, "IQGAPs: integrators of the cytoskeleton, cell adhesion machinery, and signaling networks," Cell Motility and the Cytoskeleton, vol. 55, no. 3, pp. 147-155, 2003.

[25] T. Watanabe, S. Wang, and K. Kaibuchi, "IQGAPs as key regulators of actin-cytoskeleton dynamics," Cell Structure and Function, vol. 40, no. 2, pp. 69-77, 2015.

[26] J. Noritake, T. Watanabe, K. Sato, S. Wang, and K. Kaibuchi, "IQGAP1: a key regulator of adhesion and migration," Journal of Cell Science, vol. 118, no. 10, pp. 2085-2092, 2005.

[27] C. D. White, H. H. Erdemir, and D. B. Sacks, "IQGAP1 and its binding proteins control diverse biological functions," Cellular Signalling, vol. 24, pp. 826-834, 2012.

[28] M. Chircop, "Rho GTPases as regulators of mitosis and cytokinesis in mammalian cells," Small GTPases, vol. 5, no. 2, p. e29770, 2014.

[29] A. Bielak-Zmijewska, A. Kolano, K. Szczepanska, M. Maleszewski, and E. Borsuk, "Cdc 42 protein acts upstream of IQGAP1 and regulates cytokinesis in mouse oocytes and embryos," Developmental Biology, vol. 322, no. 1, pp. 21-32, 2008.

[30] J. B. Wang, R. Sonn, Y. K. Tekletsadik, D. Samorodnitsky, and M. A. Osman, "IQGAP1 regulates cell proliferation through a novel CDC42-mTOR pathway," Journal of Cell Science, vol. 122, no. 12, pp. 2024-2033, 2009.

[31] C. McKenzie, Z. I. Bassi, J. Debski et al., "Cross-regulation between Aurora B and citron kinase controls midbody architecture in cytokinesis," Open Biology, vol. 6, no. 3, p. 160019, 2016.

[32] K. Gao, Y. Zhang, Q. Shi et al., "iASPP-PP1 complex is required for cytokinetic abscission by controlling CEP55 dephosphorylation," Cell Death \& Disease, vol. 9, no. 5, p. $528,2018$.

[33] C. Stefani, A. Lacy-Hulbert, and T. Skillman, "ConfocalVR: immersive visualization for confocal microscopy," Journal of Molecular Biology, vol. 430, no. 21, pp. 4028-4035, 2018.

[34] X. Yan, L. Cao, Q. Li et al., "Aurora C is directly associated with survivin and required for cytokinesis," Genes to Cells, vol. 10, no. 6, pp. 617-626, 2005.

[35] S. B. Thoresen, C. Campsteijn, M. Vietri et al., "ANCHR mediates Aurora-B-dependent abscission checkpoint control through retention of VPS4," Nature Cell Biology, vol. 16, no. 6, pp. 550-560, 2014.

[36] M. Adachi, A. Kawasaki, H. Nojima, E. Nishida, and S. Tsukita, "Involvement of IQGAP family proteins in the regulation of mammalian cell cytokinesis," Genes to Cells, vol. 19, no. 11, pp. 803-820, 2014.

[37] A. T. Lian, P. G. Hains, B. Sarcevic, P. J. Robinson, and M. Chircop, "IQGAP1 is associated with nuclear envelope reformation and completion of abscission," Cell Cycle, vol. 14, no. 13, pp. 2058-2074, 2015.

[38] M. Ghosh, R. Lo, I. Ivic et al., "CD13 tethers the IQGAP1ARF6-EFA6 complex to the plasma membrane to promote ARF6 activation, $\beta 1$ integrin recycling, and cell migration," Science Signaling, vol. 12, no. 579, article eaav5938, 2019.

[39] M. K. Akula, M. X. Ibrahim, E. G. Ivarsson et al., "Protein prenylation restrains innate immunity by inhibiting Rac 1 effector interactions," Nature Communications, vol. 10, no. 1, pp. 1-13, 2019.

[40] H.-C. Chuang, C.-C. Chang, C.-F. Teng et al., "MAP4K3/GLK promotes lung cancer metastasis by phosphorylating and activating IQGAP1," Cancer Research, vol. 79, no. 19, pp. 49784993, 2019. 